\title{
Lung cells support osteosarcoma cell migration and survival
}

Shibing Yu', Mitchell Stephen Fourman ${ }^{1}$, Adel Mahjoub², Jonathan Brendan Mandell', Jared Anthony Crasto', Nicholas Giuseppe Greco ${ }^{1}$ and Kurt Richard Weiss ${ }^{1,3^{*}}$

\begin{abstract}
Background: Osteosarcoma (OS) is the most common primary bone tumor, with a propensity to metastasize to the lungs. Five-year survival for metastatic OS is below 30\%, and has not improved for several decades despite the introduction of multi-agent chemotherapy. Understanding OS cell migration to the lungs requires an evaluation of the lung microenvironment. Here we utilized an in vitro lung cell and OS cell co-culture model to explore the interactions between OS and lung cells, hypothesizing that lung cells would promote OS cell migration and survival. The impact of a novel anti-OS chemotherapy on OS migration and survival in the lung microenvironment was also examined.
\end{abstract}

Methods: Three human OS cell lines (SJSA-1, Saos-2, U-2) and two human lung cell lines (HULEC-5a, MRC-5) were cultured according to American Type Culture Collection recommendations. Human lung cell lines were cultured in growth medium for $72 \mathrm{~h}$ to create conditioned media. OS proliferation was evaluated in lung co-culture and conditioned media microenvironment, with a murine fibroblast cell line (NIH-3 T3) in fresh growth medium as controls. Migration and invasion were measured using a real-time cell analysis system. Real-time PCR was utilized to probe for Aldehyde Dehydrogenase (ALDH1) expression. Osteosarcoma cells were also transduced with a lentivirus encoding for GFP to permit morphologic analysis with fluorescence microscopy. The anti-OS efficacy of Disulfiram, an ALDH-inhibitor previously shown to inhibit OS cell proliferation and metastasis in vitro, was evaluated in each microenvironment.

Results: Lung-cell conditioned medium promoted osteosarcoma cell migration, with a significantly higher attractive effect on all three osteosarcoma cell lines compared to basic growth medium, 10\% serum containing medium, and $\mathrm{NIH}-3 \mathrm{~T} 3$ conditioned medium $(p<0.05)$. Lung cell conditioned medium induced cell morphologic changes, as demonstrated with GFP-labeled cells. OS cells cultured in lung cell conditioned medium had increased alkaline phosphatase staining.

Conclusions: Lung endothelial HULEC-5a cells are attractants for OS cell migration, proliferation, and survival. The SJSA-1 osteosarcoma cell line demonstrated greater metastatic potential than Saos-2 and U-2 cells. ALDH appears to be involved in the interaction between lung and OS cells, and ALP may be a valuable biomarker for monitoring functional OS changes during metastasis.

Keywords: Osteosarcoma, Metastasis, Lung microenvironment, Disulfiram, Aldehyde dehydrogenase

\footnotetext{
* Correspondence: WeiskR@upmc.edu

'Department of Orthopaedic Surgery, University of Pittsburgh, Pittsburgh, PA, USA

${ }^{3}$ Cancer Stem Cell Laboratory, Department of Orthopaedic Surgery, 450

Technology Dr, Pittsburgh, PA 15219, USA

Full list of author information is available at the end of the article
} 


\section{Background}

Osteosarcoma (OS) is the most common primary bone tumor, with most new cases diagnosed in the first two decades of life [1, 2]. OS has a high propensity for metastasis, and over $90 \%$ of OS metastases are to the lungs [3]. The 5-year survival of patients with primary OS is about $70 \%$. However, the survival of metastatic OS has been below $30 \%$ for several decades, despite the introduction of multi-agent chemotherapy [4]. Much of this stagnation relates to our continued lack of understanding of the mechanisms of how OS cells migrate to the lungs, and what properties of the lung microenvironment are ideal for OS development and proliferation. Understanding the metastatic milieu is important to the development of novel strategies to inhibit OS metastases, and may lead to improved treatment outcomes for patients who already have metastatic OS.

It is believed that OS cells flourish in the lung microenvironment because of its high degree of vascularization and oxygenation. However, the pulmonary microenvironment is not unique in these traits. Prior work suggests that the specific interaction between OS and lung cells is the major determinant of three general fates of metastatic cells: proliferation, quiescence or apoptosis. However, our ability to regulate the OS-microenvironment to direct cells to anti- or pro-metastatic outcomes remains limited.

Here we describe an in vitro lung and OS cell co-culture model to explore the interactions between these cell types. We hypothesize that lung cells promote OS cell migration and survival.

\section{Methods}

\section{Cell lines and culture}

Three human osteosarcoma (OS) cell lines, SJSA-1, Saos2, and U-2 OS were purchased from American Type Culture Collection (ATCC) and cultured according to ATCC's recommendations. Two human lung cell lines, HULEC-5a and MRC-5, were also purchased from ATCC and cultured according to their recommendations.

\section{OS cell migration and proliferation}

HULEC-5a and MRC-5 lines were cultured in growth medium for $72 \mathrm{~h}$. The conditioned media (CM) was collected and centrifuged at $2000 \mathrm{rpm}$ for $5 \mathrm{~min}$. Each well of a 24-well plate received $600 \mu \mathrm{l}$ CM. A total of $1 \times 10^{4}$ OS cells were re-suspended in $100 \mu \mathrm{l}$ basic DMEM (without supplements), and loaded into a Corning Transwell permeable support with a pore size of $8 \mu \mathrm{m}$, which was then placed into each well of the dish. After $48 \mathrm{~h}$, the Transwell supports were removed and the migrated cells were quantified. CM from the murine fibroblast cell line NIH-3 T3 and fresh growth medium were utilized as controls. Proliferation was measured by co-culturing cells for $72 \mathrm{~h}$, then removing the Transwell support and changing the media to standard growth medium. Cells were allowed to proliferate for 10 days, then fixed and Giemsa stained.

\section{Real-Time OS cell migration and invasion}

HULEC-5a and MRC-5 CM were harvested as above. Utilizing the $x$ Celligence Real-Time Cell Analysis system (Acea Biosciences, Inc., San Diego, CA) real-time cell migration was measured using CIM-plates seeded with $2 \times 10^{4}$ OS cells in $100 \mu \mathrm{l}$ of serum free media. Readings were taken every 15 min for 100 cycles and cell index was plotted at different time points. The Cell Index is defined as $(R n-R b) / 15$, where $R n$ is the cell-electrode impedance of the well when it contains cells and $\mathrm{Rb}$ is the background impedance of the well with the media alone. OS cell invasion assays were created in a similar manner, with 3\% Corning Matrigel used to pre-coat the upper chamber.

\section{OS and lung direct co-culture}

OS cells were stably transduced with a lentivirus encoding for green fluorescent protein (GFP). A total of $2 \times 10^{4}$ $\mathrm{OS}_{\mathrm{GFP}}$ cells were seeded in a 24-well plate. These same wells were seeded with either $2 \times 10^{4}$ HULEC-5a or MRC- 5 cells. After $72 \mathrm{~h}$, cell morphology was visualized by fluorescence microscope.

\section{Alkaline phosphatase (ALP) staining}

A co-culture of $2 \times 10^{4}$ OS cells and $2 \times 10^{4}$ HULEC-5a was performed for 24, 48 and $72 \mathrm{~h}$. Multiple time points were included to determine if ALP expression was different between each group at a given time. At each time point, cells were fixed and stained for ALP expression using SigmaFAST BCIP/NBT (Sigma-Aldrich Co LLC, USA). Similar to the direct OS and lung cell co-culture, OS cells were also cultured in CM from HULEC-5a for $72 \mathrm{~h}$ and stained for ALP.

\section{Real-time PCR}

SJSA-1 and Saos- 2 cells ( $1 \times 10^{5}$ each) were cultured in growth media or HULEC-5a CM for $48 \mathrm{~h}$. Total RNA was harvested using Ambion Trizol Reagent (ThermoFisher Scientific, USA). RNA ( $1 \mu \mathrm{g})$ was utilized for cDNA using Applied Biosystems High Capacity cDNA kit (ThermoFisher Scientific, USA). A total of 8 ng of cDNA was used as template and PCR was run on an Applied Biosystems StepOne Real-Time PCR Thermocycler (ThermoFisher Scientific, USA). ALDH1 primer sequence was forward: 5'-CCTGTCCTACTCACCGATTTG-3' and reverse: 5'CCTCCTCAGTTGCAGGATTAAA-3'.

\section{Disulfiram treatment}

Disulfiram (Sigma-Aldrich Co LLC, USA) was dissolved in DMSO and in working concentrations of 10, 50, 100, 200 and $500 \mathrm{nM}$ in growth medium or HULEC-5a CM. 
In the CM culture group, $2 \times 10^{4}$ SJSA- 1 or Saos- 2 cells were seeded in each well of a 24-well plate for $24 \mathrm{~h}$. In the co-culture group, $2 \times 10^{4}$ HULEC cells together with $2 \times 10^{4}$ SJSA-1 or Saos- 2 cells were seeded in each well of a 24well plate for $24 \mathrm{~h}$. This was followed by adding fresh growth media containing disulfiram and culturing for another $72 \mathrm{~h}$. Cells were then fixed and stained for ALP.

\section{5-Bromo-2'-deoxyuridine (BrdU) staining}

A $10 \mathrm{mM}$ stock solution of BrdU (Sigma-Aldrich Co LLC, USA) was diluted 1:1000 in growth medium or HULEC CM. SJSA- 1 or Saos- 2 cells $\left(2 \times 10^{4}\right)$ were seeded in a 24-well plate for $24 \mathrm{~h}$. Cell medium was changed to BrdU-containing medium for another $4 \mathrm{~h}$. A BrdU staining kit was used for immunohistochemistry (ThermoFisher Scientific, USA).

\section{Terminal deoxynucleotidyl transferase-mediated dUTP} nick end-labeling (TUNEL) assay

OS Cells were grown in 24-well plates at a seeding density of $2 \times 10^{4}$ cells-per-well in growth media or HULECCM for $48 \mathrm{~h}$. TUNEL assay was carried out using ApoptTag Peroxidase In Situ Apoptosis Detection Kit (EMD Millipore, Billerica, MA, USA).

\section{Statistical analysis}

Data was analyzed using Prism 7.0 (GraphPad, La Jolla, CA, USA). Multi-group analysis was performed using analysis of variance with Tukey's post-test for between-group comparisons. Two-group analysis was performed using $t$ tests for parametric data and the Mann-Whitney $U$ test for non-parametric distributions. In all cases, $p<0.05$ was considered significant. Values were expressed as mean \pm standard deviation.

\section{Results}

\section{Lung cell conditioned medium (CM) induces OS cells} migration

To evaluate if different types of lung cells are variably attractive to different OS cell lines, we used three OS cell lines: Saos-2, SJSA-1 and U-2 OS, and two lung cell lines, HULEC-5a and MRC-5, to perform Transwell experiments. After $48 \mathrm{~h}, \mathrm{HULEC}-5 \mathrm{a} \mathrm{CM}$ had a significantly higher $(p<0.05)$ attractive effect on all three OS cell lines compared to basic growth medium, 10\% serum containing medium and NIH3T3 CM. Among these three OS cell-lines, Saos-2 cell had the highest and SJS11 the lowest migration in HULEC-5a CM (Fig. 1a). MRC-5 CM was more attractive to all three OS cell lines compared to basic medium and $10 \%$ serum containing medium, but not to NIH3T3 CM (Fig. 1a). To further evaluate the dynamic migration of these three OS cell lines in HULEC-5a CM and MRC-5 CM, we repeated the migration experiment using the $\mathrm{xCelligence} \mathrm{Real-}$
Time Cell Analysis system. HULEC-5a CM had a significantly higher attractive effect on all three OS cell lines. Moreover, real-time migration data reveals that SJSA-1 cell migration in HULEC-5a CM peaks at $5 \mathrm{~h}$, $\mathrm{U}-2$ OS cell migration peaks at $15 \mathrm{~h}$ and Saos-2 migration peaks at $20 \mathrm{~h}$ (Fig. 1b). Migration peaks were determined by assessing the point at which the slope of the curve was greatest.

\section{Lung cells further increase OS cells migration}

To dissect the role of lung cells in the attraction of OS cell migration, we investigated Saos- 2 and SJSA-1 cells migration in HULEC-5a CM containing $1 \times 10^{4}$ or $5 \times 10^{4}$ HULEC cells. We found that $1 \times 10^{4}$ and $5 \times 10^{4}$ HULEC-5a cells, when in the bottom well, increased SJSA-1 cell migration compared to HULEC-5a CM in the bottom well. Interestingly, Saos- 2 migration in $1 \times 10^{4}$ HULEC-5a cells is significantly higher than in $5 \times 10^{4}$ HULEC-5a cells or CM (Fig. 2a). SJSA-1 cell migration started earlier than Saos-2 in both HULEC-5a cells and CM (Fig. 2a). When evaluating SJSA-1 migration in MRC-5 cells and CM, we found that $1 \times 10^{4}$ MRC-5 cells led to SJSA-1 cell migration, whereas MRC-5 CM and 5x10 $0^{4}$ cells had lower migration rates than $1 \times 10^{4}$ cells (Fig. 2b).

\section{Lung cell CM stimulates OS cell morphology change and invasion}

We used a lentivirus encoding for GFP to label the Saos-2, SJSA-1 and U-2 OS cell lines (Fig. 3a, column 1, 3, 5). Bright field pictures were taken of the same area (Fig. 3a, column 2, 4, 6). We used several parameters to assess morphologic changes in OS cells in the OS and lung cell co-culture. Gross morphologic difference is measured by a systematic difference in two-dimensional projected area. Our results showed that Saos-2 and SJSA-1 cells have a significantly larger projected area when in co-culture with HULEC-5a or MRC-5 cells compared to isolated Saos-2 and SJSA-1 culture alone (Fig. 3a, columns 1 and 3). U-2 OS cells demonstrated the opposite trend, and had a significantly smaller projected area in co-culture with HULEC-5a and MRC-5 cells compared to U-2 OS cells alone (Fig. 3a, column 5). Cell shape assesses roundness vs elongation. Similar to changes in projected area, Saos-2 and SJSA-1 cells showed greater elongation and less roundness when in co-culture with HULEC-5a or MRC-5 cells compared to isolated Saos-2 and SJSA-1 culture (Fig. 3a, columns 1 and 3). U-2 OS again displayed the opposite trend, showing reduced elongation but more roundness when in co-culture with HULEC-5a and SISA1 cells compared to U-2 OS alone (Fig. 3a, column 5).

Considering the cytoskeletal morphologic changes during the acquisition of OS invasive capacity [5-7], we assessed OS invasion in 3\% Matrigel to HULEC-5a CM and MRC-5 CM, again with the xCELLigence Real-Time 


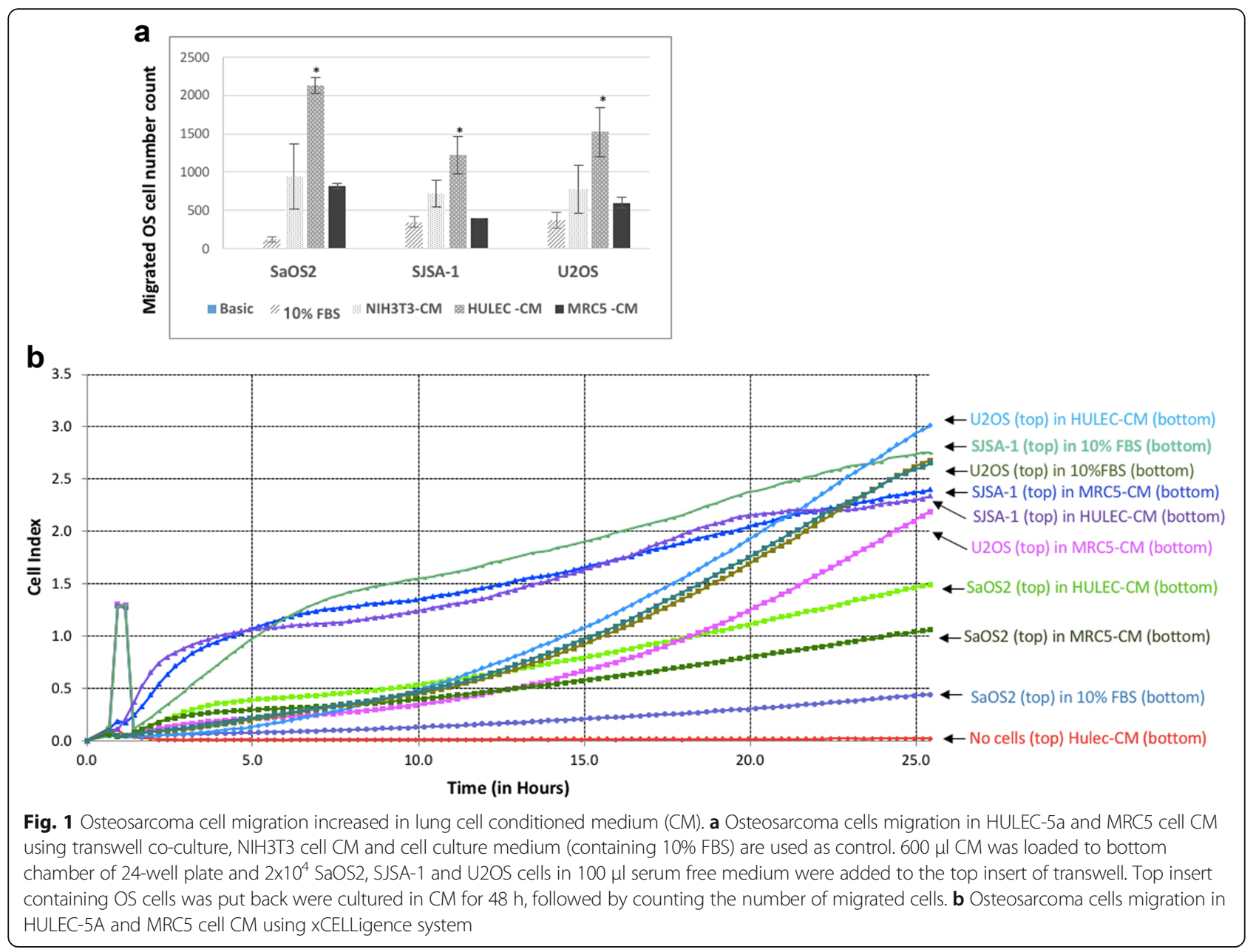

Cell Analysis system. SJSA-1 had a significantly higher invasion capacity in the presence of HULEC-5a CM. In contrast, U-2 OS cell invasiveness was relatively lower, and Saos- 2 cell migration was completely blocked by Matrigel (Fig. 3b).

\section{Lung cells induce ALP expression in OS cell lines}

Alkaline Phosphatase (ALP) has been long appreciated to correlate with active bone metabolism, and high ALP levels correlate with OS metastases [8-11]. In addition to morphologic changes, the OS metastatic phenotype correlates with alkaline phosphatase (ALP) staining in OS cells that have also changed in the presence of HULEC-5a coculture. ALP-positive staining appeared after $24-48 \mathrm{~h}$ in Saos- 2 and SJSA- 1 co-culture with HULEC-5a, while ALP expression in Saos-2 and SJSA-1 cells was undetectable at $24 \mathrm{~h}$ and slightly increased at $48 \mathrm{~h}$ (Fig. 4a, b, c). At $72 \mathrm{~h}$, ALP staining in Saos-2 and SJSA-1 in co-culture with HULEC-5a was still significantly greater than from Saos-2 or SJSA-1 cells alone (Fig. 4a, b, c). ALP in U-2 OS and HULEC-5a cells was undetectable at 24, 48 and $72 \mathrm{~h}$ (Fig. 4a, b, c). To determine if HULEC-5a cells are required to increase ALP expression, we used HULEC-5a $\mathrm{CM}$ in the co-culture and analyzed ALP expression in Saos-2, SJSA-1 and U-2 OS. There was no significant difference in ALP expression between regular medium and CM (Fig. 4d, e, f).

\section{Lung cell supported migrated cell survival}

The survival of migrating OS cells is a critical step in OS metastasis. To explore the effects of lung cells on migrated OS cell survival, we induced Saos-2, SJSA-1 and U-2 OS cell migration in HULEC-5a and MRC-5 $\mathrm{CM}$ for $72 \mathrm{~h}$, then switched to regular culture medium for an additional 10 days. Compared to migrated Saos-2 and SJSA-1 cells, migrated U-2 OS cells had the most colony growth in regular medium, HULEC-5a CM and MRC-5 CM. Migrated SJSA-1 cells grew in HULEC-5a CM, but not MRC-5 CM. Migrated Saos- 2 cells were not observed in HULEC-5a CM, MRC-5 CM and control media (Fig. 5a, b). To uncover the mechanisms underlying increased cell migration, we assessed migrated OS cell proliferation and found that HULEC-5a CM benefited SJSA-1 cell proliferation (Fig. 5c). 


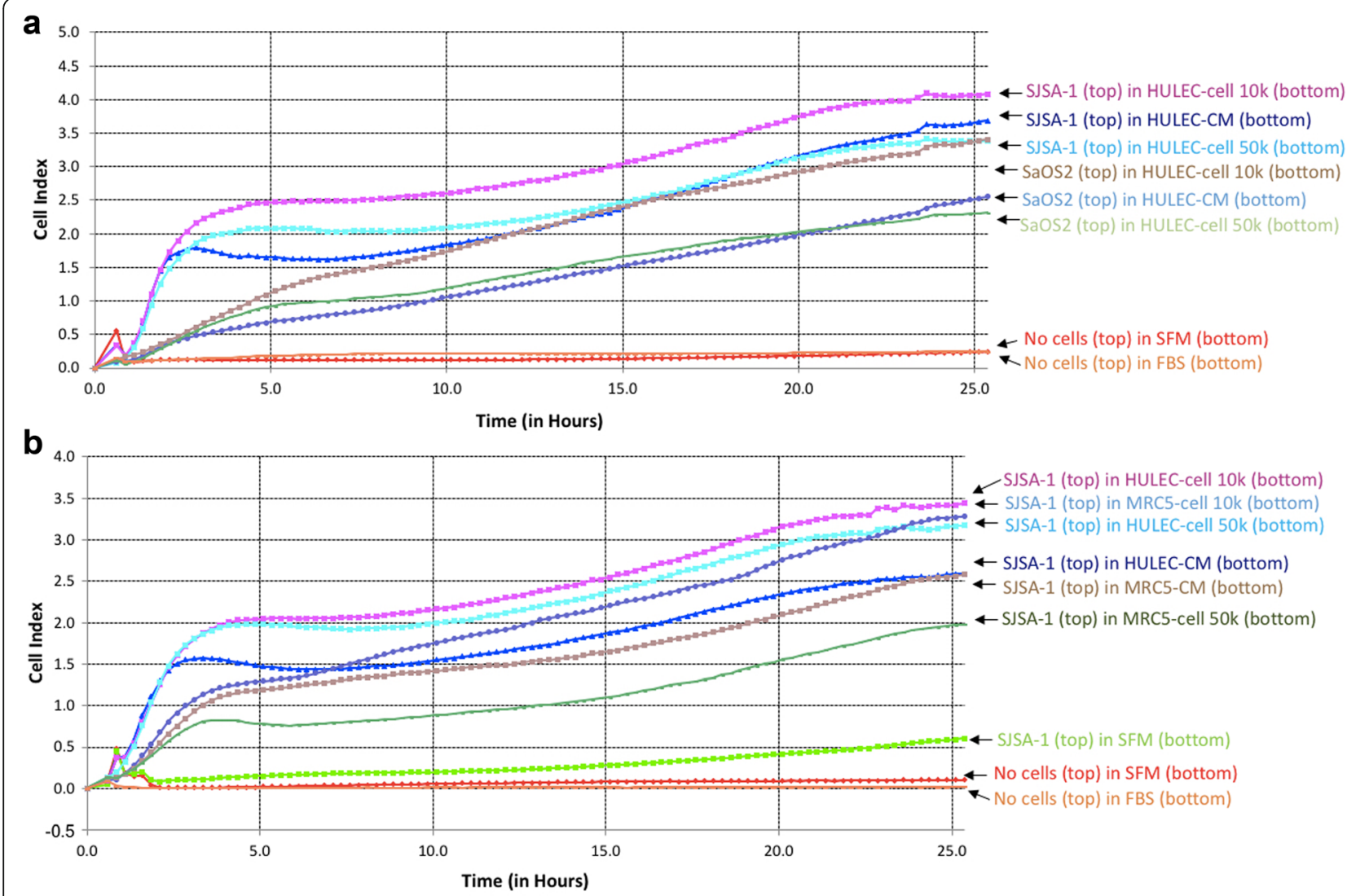

Fig. 2 Osteosarcoma cells migration to lung cell CM were further increased in the presence of lung cells that were placed in the bottom

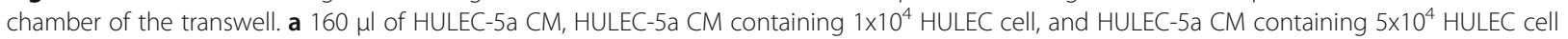
were loaded onto the bottom well of CIM Plate 16. $2 \times 10^{4}$ SaOS2 or SJSA-1 cells in $100 \mu$ l serum free medium were added to the top well of CIM Plate 16. OS cell migration index was calculated every $15 \mathrm{~min}$ for 100 reading. Each plate had negative controls where no cells were added to the top well and either serum free media or FBS was added to the bottom well. b $160 \mu \mathrm{l}$ of HULEC-5a CM, HULEC-5a CM containing 1x104 HULEC cell, and HULEC-5a CM containing $5 \times 10^{4}$ HULEC cell were placed in the bottom chamber of the transwell and compared to MRC5 CM, MRC5 CM containing $1 \times 10^{4}$ MRC5 cell and MRC5 CM containing $5 \times 10^{4}$ MRC5 loaded onto the bottom well of CIM-Plate $16.2 \times 10^{4}$ SJSA-1 cells in $100 \mu$ l serum free medium were added to top well of CIM Plate 16, and SJSA-1 cell migration index was calculated every 15 min for 100 reading. Each plate had negative controls where no cells were added to the top well and either serum free media or FBS was added to the bottom well. Serum free media in the bottom well was also tested with SJSA cells

\section{ALDH is involved in the process of lung: OS cell interaction}

Akdehyde dehydrogenase (ALDH) mRNA level in Saos-2 and SJSA-1 cells was elevated after being cultured in HULEC-5a CM, at a fold change of 9 and 22, respectively (Fig. 6a). To further investigate the role of this cancer stem cell marker in the interaction between lung and OS cells, we treated co-cultured cells with disulfiram, an ALDH-specific inhibitor, at doses of 10, 50, 100, 200 and $500 \mathrm{nM}$ for $72 \mathrm{~h}$. We found that ALP staining of Saos-2 cells was significantly suppressed in a dose dependent manner, in particular at a dose of $100 \mathrm{nM}$ and higher. Moreover, HULEC-5a cells reversed the disulfirammediated suppression of ALP in Saos- 2 cells at $100 \mathrm{nM}$, but not at higher disulfiram doses of 200 and $500 \mathrm{nM}$ (Fig. 6b). HULEC-5a CM did not affect the disulfirammediated suppression of ALP in Saos-2 cells (Fig. 6b).
ALP expression of SJSA-1 was also significantly inhibited by disulfiram in a dose-dependent manner. HULEC-5a cells attenuated the suppression of ALP expression in SJSA-1 cells even at higher doses of disulfiram (500nM). The effect of HULEC-5a CM on the ALP expression of SJSA-1 cells is similar to HULEC-5a cells (Fig. 6b). On the contrary, MRC-5 cells did not influence the disulfirammediated inhibition of ALP expression in Saos- 2 and SJSA-1 cells (Fig. 6b, d). Interestingly, HULEC-5a CM increases Saos-2 and SJSA-1 cell proliferation in the presence of $100 \mathrm{nM}$ disulfiram for $48 \mathrm{~h}$ (Fig. 6c).

\section{Discussion}

OS is the most common primary malignant bone tumor, with peak incidence rates during adolescence [12-14]. Pulmonary OS metastasis is the predominant determinant of disease mortality [4]. However, the mechanisms 


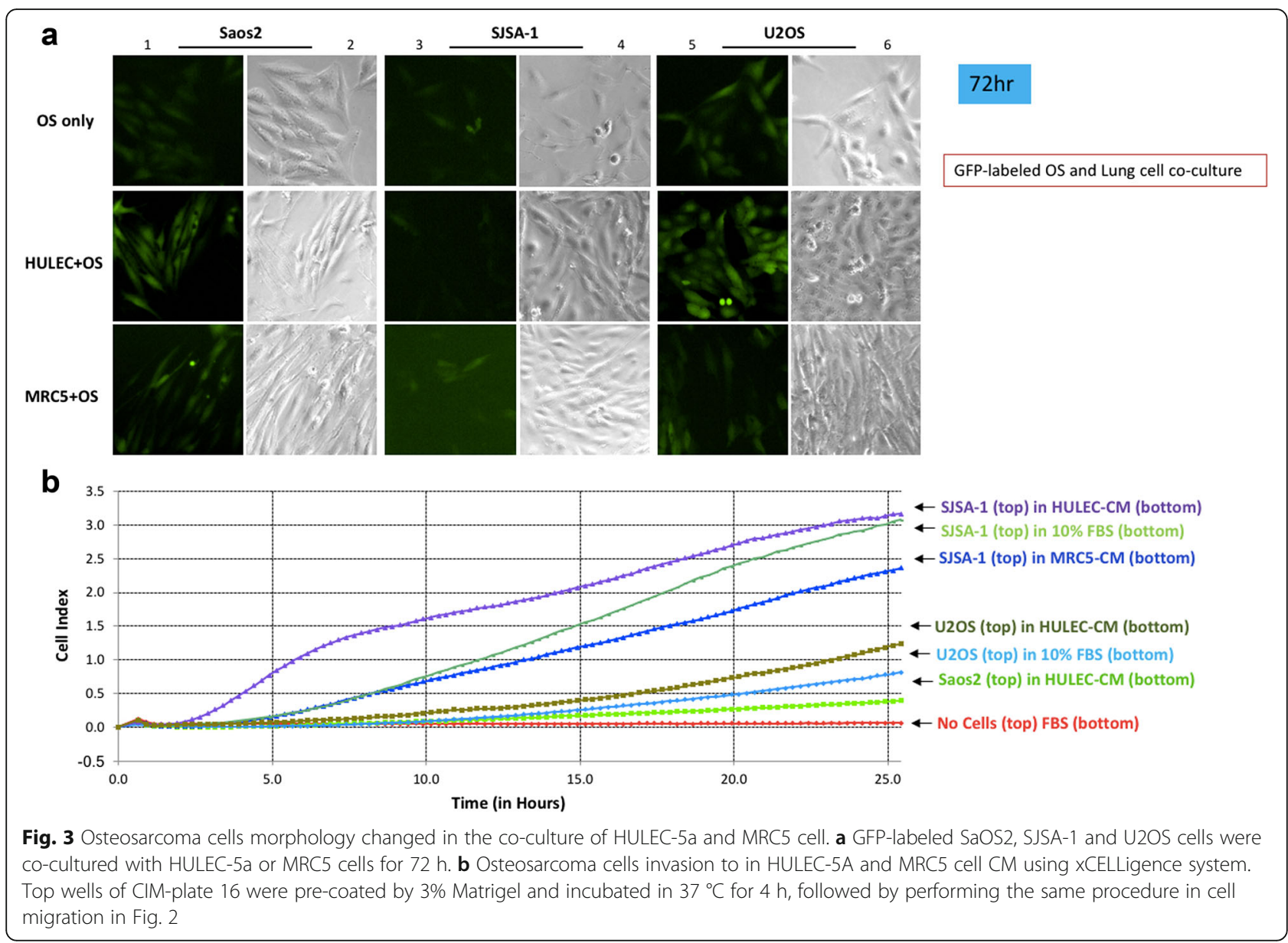

underlying OS cell migration to the lungs and their interaction with lung cells remain poorly understood. Here we found that different OS cell sub-types have different capacities for migration in response to the same human lung cell-line. The SJSA-1 cell-line, a so-called fibroblastic OS cell-line with a high metastatic potential, had significantly greater migration and metastasis marker gene expression in response to HULEC-5a cell exposure compared to Saos-2 and U-2 OS cells, two epithelial OS cell-lines. Further, different types of lung cells have different attractive potential. SJSA-1 migration and invasion in the presence of HULEC-5a, a lung endothelial cell-line, is significantly greater than in the presence of MRC-5, a fibroblastic lung cell-line.

\section{Heterogeneity of OS cell with different metastatic potential has different response to lung cell environment: cell or cell-conditioned medium}

The SJSA-1 cell line has been used as an OS metastatic model in several prior studies [15-19], and Saos-2 and U-2 OS cells have been found to have an undetectable metastatic effect $[16,19]$. We therefore used SJSA-1 as our pro-metastatic cell model, and Saos-2 and U-2 OS as controls. In line with previous reports, our data demonstrated that SJSA-1 has a significantly higher migration potential when exposed to HULEC-5a CM compared to control medium (Figs. 1a, b, 2a, b, 5a and b). Although Saos- 2 cells have a slightly higher migration rate than SJSA-1 cells (Fig. 1b), Saos-2 cells cannot survive in a novel microenvironment (Fig. 5a and b). U-2 OS cells have similar migration and survival capabilities to SJSA-1 cells when exposed to HULEC-5a CM (Figs. 1a, 5a and b). However, U-2 OS cells rarely invade through the basement membrane of distant tissue compared to SJSA-1 cells (Fig. 3b). It is well-accepted that cancer metastasis involves several critical stages, including tumor invasion through the local basement membrane, tumor cell survival within the bloodstream and the initial survival of tumor cells in the distant tumor stromal environment [20, 21]. SJSA-1 has dramatically higher invasion (Fig. 3b), migration (Fig. 1a and b), survival and proliferation potential in lung cell co-culture (Fig. 5a and b). This reflects the high metastatic potential of SJSA-1 in animal models.

An interesting finding in our study was the different invasion and migration potential of OS cells following lung cell co-culture. SJSA-1 had a higher invasiveness 

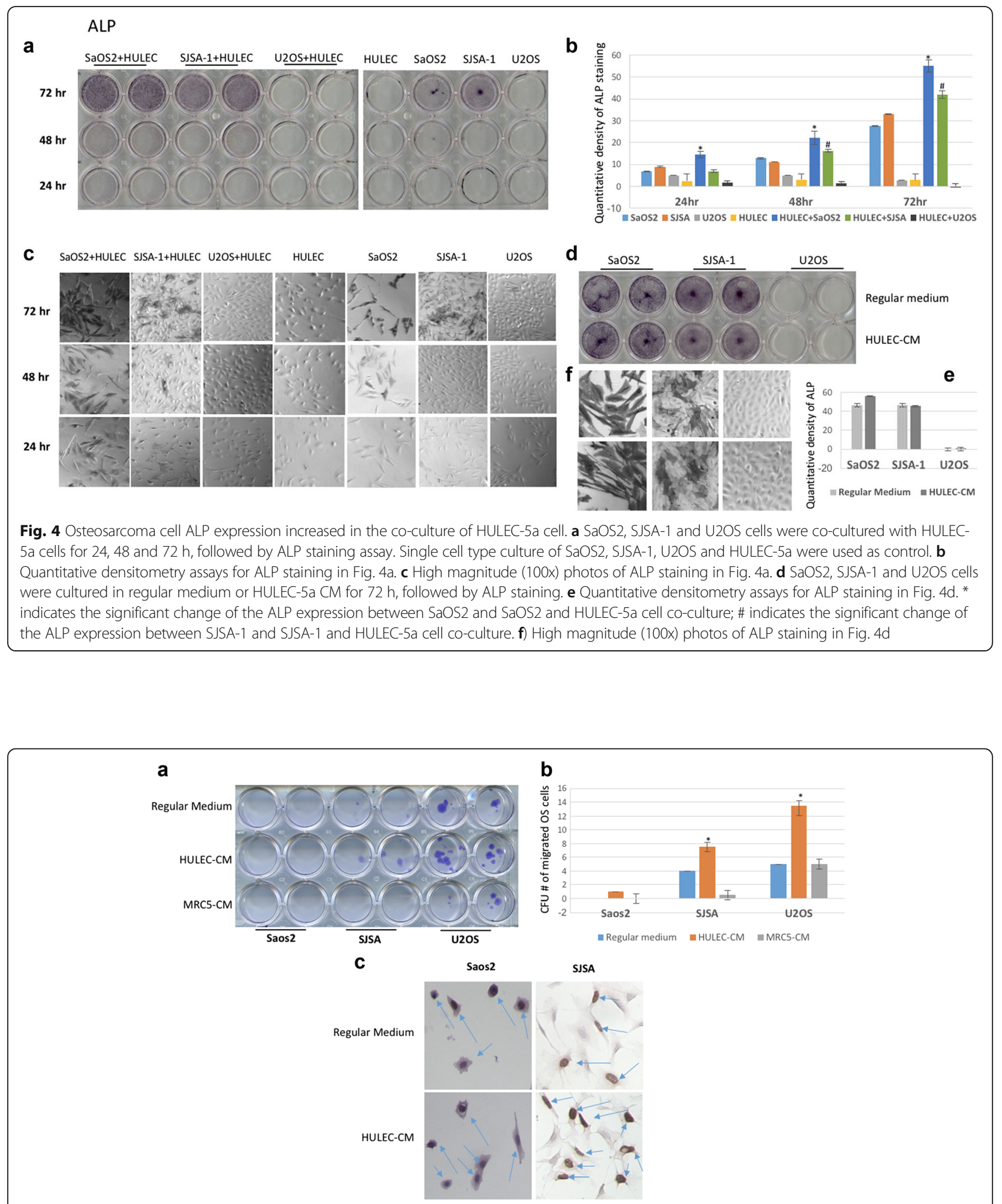

BrdU staining

Fig. 5 Migrated Osteosarcoma cells proliferation and survival increased in the HULEC-5a CM. a SaOS2, SJSA-1 and U2OS cells were induced to migrate to HULEC-5a or MRC5 CM for $48 \mathrm{~h}$, followed by culturing the migrated OS cells in regular culture medium for another 10 days. OS cells were fixed and visualized by Giemsa staining. OS cell migration in regular medium is used as control. b Quantitative assays for Giemsa staining positive colony of the OS cell in Fig. 5a. c BrdU staining of the SaOS2 and SJSA-1 cells migrating to HULEC-5a and MRC5 CM. Brown color represents the positive staining 


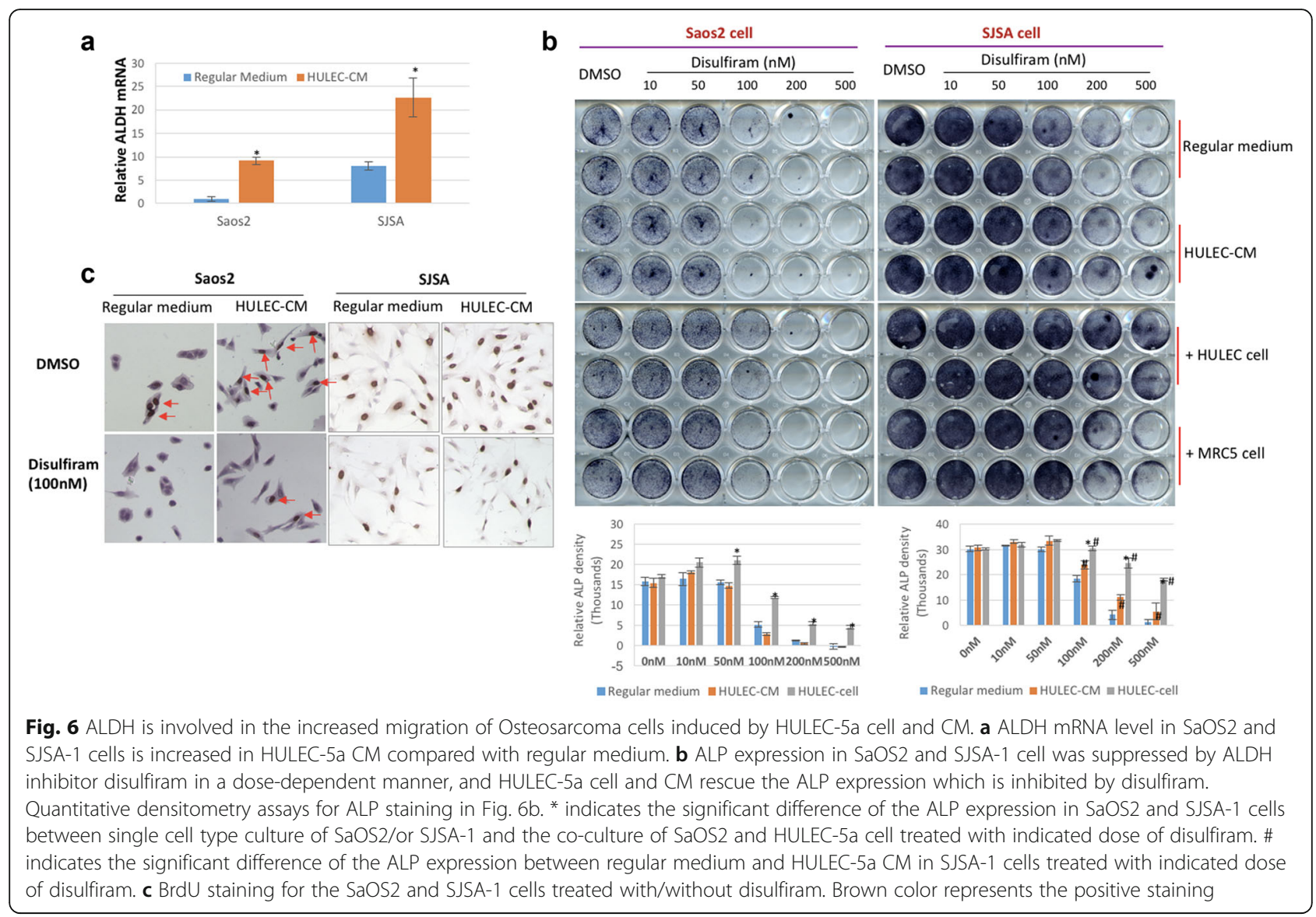

(Fig. 3b) but relatively lower migration potential (Fig. 1b) compared to cells grown in HULEC-5a CM. Saos-2 and U-2 OS have higher migration (Fig. 1a), but reduced invasion potential compared to cells grown in HULEC5a CM (Fig. 3b). To migrate, the cell body must modify its shape and stiffness to interact with the surrounding tissue structures. Invasion requires adhesion, proteolysis of extracellular matrix components and migration [22]. SJSA-1 cells become polarized and elongate when cocultured with HULEC-5a cells (Fig. 3a), an alteration that may be a sentinel event of cell invasion. In contrast, U-2 OS cell morphology does not change when co-cultured with HULEC-5a cells (Fig. 3a).

\section{Different lung cell types have different effects on OS cell migration}

Lung tissue is made up of multiple cell types, including epithelial, endothelial and fibroblastic cells. To characterize which cell type most effectively attracts OS cells, we used CM from HULEC-5a, an endothelial cell-line, and MRC-5, a fibroblastic cellline. Our data showed that HULEC-5a CM is a more efficient attractant for OS cell migration compared to MRC-5 CM (Figs. 1b, b, 2a and b).
To further investigate if the lung cells themselves are required to stimulate OS migration, we used CM containing doses of HULEC-5a cells or MRC- 5 cells. Our data demonstrate that $1 \times 10^{4}$ HULEC-5a or MRC-5 cells further stimulate SJSA-1 cell migration compared to HULEC-5a CM alone (Fig. 2a and b). This suggests that some unknown properties of HULEC-5a and MRC-5 cells also play important roles in the promotion of OS cell migration. Interestingly, $5 \times 10^{4}$ HULEC- 5 a or MRC- 5 cells have the same effect as CM controls (Fig. 2a and b). The reason why higher doses of HULEC-5a and MRC- 5 cells have a reduced effect on SJSA-1 cell migration remains unclear. One possible explanation may be because too many cells are competing for available nutrients in vitro and producing cytotoxic byproducts.

\section{ALP activity is a marker for OS lung metastasis}

ALP has been previously demonstrated to be an indicator of OS metastasis in multiple clinical studies [8-11]. High serum ALP levels are associated with the presence of metastatic OS at the time of diagnosis, and poor disease prognosis. Serum ALP level is a convenient and effective biomarker of OS prognosis. In our study we used ALP expression to monitor OS cell functional changes in response to culture with HULEC-5a cells or 
CM. We found that ALP expression in SJSA-1 and Saos-2 cells was dramatically increased by HULEC-5a cell coculture. ALP expression was also noted at earlier time points (Fig. 4a, b and c). In contrast, ALP expression was not influenced by HULEC-5a CM (Fig. 4d, e and f). This suggests that functional changes to $\mathrm{OS}$ cells requires direct interaction with lung cells.

\section{ALDH is involved in the process of OS cell migration to lung cell}

ALDH is a biomarker for cancer stem cells [23, 24].

Previous studies by our group have shown that ALDH activity is greater in the highly metastatic K7M2 OS cellline than in non-metastatic K12 OS cells. We have also correlated ALDH activity with clinical OS metastasis, suggesting that ALDH may be a therapeutic target specific to OS cells with high metastatic potential [25]. Subsequent studies found that Retinal, the precursor to retinoic acid with known antitumor properties, targets ALDH-positive cancer stem cells and alters the phenotype of highly metastatic OS cells [26]. When determining if ALDH was involved in OS cell functional changes in response to the HULEC-5a microenvironment, we found that HULEC-5a CM dramatically increases ALDH expression in SJSA-1 and Saos-2 cells (Fig. 6a). In contrast, in our loss-of-function experiments we treated our experimental groups with doses of disulfiram, an ALDH inhibitor. Our findings demonstrated that HULEC-5a cells help SJSA-1 to resist disulfiram inhibition and maintain their pro-metastatic function, indicated by their preserved ALP activity (Fig. 6b). HULEC5 a cells also supported SJSA-1 cell survival and proliferation in the presence of disulfiram (Fig. 6c). Our current results are consistent with our previous findings [25, 26] as well as those of other groups [23, 24].

While the findings of this study contribute to our understanding of the mechanisms behind OS metastasis, we recognize several limitations to this work. Our sample size throughout this study was often based on single experiments, which highlights the preliminary nature of our work. Our utilization of a murine fibroblast cell line as a control was a decision made out of convenience, as our group has significant experience and success with this cell line. This will be altered in future studies. However, we believe that the relationships highlighted here would persist regardless of cell line used.

Most importantly, our findings illustrate associative relationships between OS cells and lung cells. While these relationships may serve as the basis for new theories, we have not established mechanisms (beyond ALDH activity) that explain them. While we concede that our findings do not yield mechanistic conclusions, we believe that the data presented here (1) describe a new strategy by which the mechanisms behind OS metastasis may be elucidated, and (2) suggest that lung cells per se are active participants in the metastatic process, either directly or indirectly or both. Heretofore, this relationship has been suggested by OS's clinical behavior, but not demonstrated. We hope that our findings will serve as the foundation of future work that will identify causal factors and mechanisms that will explain the provocative phenomena we report here.

\section{Conclusions}

We have demonstrated that fibroblastic SJSA-1 OS cells have a higher lung metastatic potential than epithelial Saos-2 and U-2 OS cells. Lung endothelial HULEC-5a cells are attractants for OS cell migration, proliferation and survival. Further, the cancer stem cell marker ALDH is involved in the interaction between lung and OS cells, and ALP could be a valuable biomarker for monitoring functional OS changes during the metastatic process. These data will form the basis of future work that will delve deeper into understanding the mechanisms by which metastatic OS cells and the lung microenvironment interact in the metastatic process.

\section{Abbreviations \\ ALDH: Aldehyde dehydrogenase; ALP: Alkaline phosphatase; ATCC: American type culture collection; BrdU: 5-Bromo-2'-deoxyuridine; CM: Conditioned media; GFP: Green fluorescent protein; OS: Osteosarcoma; TUNEL: Terminal deoxynucleotidyl transferase-mediated dUTP nick end-labeling}

\section{Acknowledgements}

Jessica Tebbets BS - Department of Orthopaedic Surgery, University of Pittsburgh, Pittsburgh PA J.Tebbets@pitt.edu. Dr. Weiss is a member of the University of Pittsburgh Cancer Institute (UPCI).

\section{Funding}

NIH K Award \#5K08CA177927-03, awarded to principal investigator, Dr. Kurt Richard Weiss.

\section{Availability of data and materials}

The datasets during and/or analysed during the current study available from the corresponding author on reasonable request.

\section{Authors' contributions}

SY, KW, NG composed the protocol. JM, MF, AM, NG performed the experiments. SY, KW, MF, JC, AM, NG analyzed the data. All authors contributed to writing of the manuscript. All authors read and approved the final manuscript.

\section{Competing interests}

The authors declare that they have no competing interests.

Consent for publication

Not applicable.

Ethics approval and consent to participate

No consent or ethical approval required, this is an in vitro study using commercially available cell lines.

\section{Author details}

'Department of Orthopaedic Surgery, University of Pittsburgh, Pittsburgh, PA, USA. ${ }^{2}$ School of Medicine, University of Pittsburgh, Pittsburgh, PA, USA. ${ }^{3}$ Cancer Stem Cell Laboratory, Department of Orthopaedic Surgery, 450 Technology Dr, Pittsburgh, PA 15219, USA. 
Received: 26 July 2016 Accepted: 6 January 2017

\section{Published online: 25 January 2017}

\section{References}

1. Whelan J, McTiernan A, Cooper N, Wong YK, Francis M, Vernon S, Strauss SJ. Incidence and survival of malignant bone sarcomas in England 1979-2007. Int J Cancer. 2012;131:E508-17.

2. Bielack SS, Carrle D, Hardes J, Schuck A, Paulussen M. Bone tumors in adolescents and young adults. Curr Treat Options Oncol. 2008;9:67-80.

3. Hegyi M, Semsei AF, Jakab Z, Antal I, Kiss J, Szendroi M, Csoka M, Kovacs G. Good prognosis of localized osteosarcoma in young patients treated with limb-salvage surgery and chemotherapy. Pediatr Blood Cancer. 2011:57:415-22.

4. Botter SM, Neri D, Fuchs B. Recent advances in osteosarcoma. Curr Opin Pharmacol. 2014;16:15-23.

5. Bakal C, Aach J, Church G, Perrimon N. Quantitative morphological signatures define local signaling networks regulating cell morphology. Science. 2007;316:1753-6.

6. Kirpensteijn J, Kik M, Rutteman GR, Teske E. Prognostic significance of a new histologic grading system for canine osteosarcoma. Vet Pathol. 2002;39:240-6.

7. Straw RC, Powers BE, Klausner J, Henderson RA, Morrison WB, McCaw DL, Harvey HJ, Jacobs RM, Berg RJ. Canine mandibular osteosarcoma: 51 cases (1980-1992). J Am Anim Hosp Assoc. 1996:32:257-62.

8. Levine AM, Rosenberg SA. Alkaline phosphatase levels in osteosarcoma tissue are related to prognosis. Cancer. 1979;44(6):2291-3.

9. Bacci G, Longhi A, Ferrari S, Lari S, Manfrini M, Donati D, Forni C, Versari M. Prognostic significance of serum alkaline phosphatase in osteosarcoma of the extremity treated with neoadjuvant chemotherapy: recent experience at Rizzoli institute. Oncol Rep. 2002;9(1):171-5.

10. Bacci G, Picci P, Ferrari S, Orlandi M, Ruggieri P, Casadei R, Ferraro A, Biagini $R$, Battistini A. Prognostic significance of serum alkaline phosphatase measurements in patients with osteosarcoma treated with adjuvant or neoadjuvant chemotherapy. Cancer. 1993;71(4):1224-30.

11. Ren H, Sun L, Li H, Ye Z. Prognostic significance of serum alkaline phosphatase level in osteosarcoma: a meta-analysis of published data. Biomed Res Int. 2015;2015:160835.

12. Mirabello L, Troisi RJ, Savage SA. Osteosarcoma incidence and survival rates from 1973 to 2004: data from the surveillance, epidemiology, and End results program. Cancer. 2009;115(7):1531-43.

13. Mirabello L, Troisi RJ, Savage SA. International osteosarcoma incidence patterns in children and adolescents, middle ages and elderly persons. Int J Cancer. 2009;125(1):229-34

14. Savage SA, Mirabello L. Using epidemiology and genomics to understand osteosarcoma etiology. Sarcoma. 2011;2011:548151. doi:10.1155/2011/ 548151. Epub 2011 Mar 8.

15. Ragland BD, Bell WC, Lopez RR, Siegal GP. Cytogenetics and molecular biology of osteosarcoma. Lab Invest. 2002:82:365-73.

16. Liu B, Wu Y, Zhou Y, Peng D. Endothelin a receptor antagonism enhances inhibitory effects of anti-ganglioside GD2 monoclonal antibody on invasiveness and viability of human osteosarcoma cells. PLoS One. 2014;9(4):e93576.

17. Mikami I, You L, He B, Xu Z, Batra S, Lee AY, Mazieres J, Reguart N, Uematsu K, Koizumi K, Jablons DM. Efficacy of Wnt-1 monoclonal antibody in sarcoma cells. BMC Cancer. 2005;5:53.

18. Pignochino Y, Grignani G, Cavalloni G, Motta M, Tapparo M, Bruno S, Bottos A, Gammaitoni L, Migliardi G, Camussi G, Alberghini M, Torchio B, Ferrari S, Bussolino F, Fagioli F, Picci P, Aglietta M. Sorafenib blocks tumour growth, angiogenesis and metastatic potential in preclinical models of osteosarcoma through a mechanism potentially involving the inhibition of ERK1/2, MCL-1 and ezrin pathways. Mol Cancer. 2009;8:118.

19. Mohseny AB, Machado I, Cai Y, Schaefer KL, Serra M, Hogendoorn PCW. Llombart-Bosch A and Cleton-Jansen AM. Functional characterization of osteosarcoma cell lines provides representative models to study the human disease. Lab Invest. 2011;91:1195-205.

20. Wooda SL, Pernemalm M, Crosbie PA, Whetton AD. The role of the tumormicroenvironment in lung cancer-metastasis and its relationship to potential therapeutic targets. Cancer Treat Rev. 2014;40(4):558-66.

21. Catalanoa V, Turdoa A, Francoa SD, Dieli F, Todaroa M, Stassi G. Tumor and its microenvironment: a synergistic interplay. Semin Cancer Biol. 2013;23:522-32.
22. Friedl $P$, Wolf $K$. Tumour-cell invasion and migration: diversity and escape mechanisms. Nat Rev Cancer. 2003;3:362-74.

23. Ginestier C, Hur MH, Charafe-Jauffret E, Monville F, Dutcher J, Brown M, et al. ALDH1 is a marker of normal and malignant human mammary stem cells and a predictor of poor clinical outcome. Cell Stem Cell. 2007;1:555-67.

24. Marcato P, Dean CA, Giacomantonio CA, Lee PW. Aldehyde dehydrogenase: its role as a cancer stem cell marker comes down to the specific isoform. Cell Cycle. 2011;10:1378-84.

25. Mu X, Isaac C, Schott T, Huard J, Weiss K. Rapamycin inhibits ALDH activity, resistance to oxidative stress, and metastatic potential in murine osteosarcoma cells. Sarcoma Volume. 2013;2013:480713.

26. Mu X, Patel S, Mektepbayeva D, Mahjoub A, Huard J, Weiss K. Retinal targets ALDH positive cancer stem cell and alters the phenotype of highly metastatic osteosarcoma cells. Sarcoma. 2015;2015:784954.

\section{Submit your next manuscript to BioMed Central and we will help you at every step:}

- We accept pre-submission inquiries

- Our selector tool helps you to find the most relevant journal

- We provide round the clock customer support

- Convenient online submission

- Thorough peer review

- Inclusion in PubMed and all major indexing services

- Maximum visibility for your research

Submit your manuscript at www.biomedcentral.com/submit
Biomed Central 